\title{
Sexual medicine disparities between Asia and North America: commentary on male sexual dysfunction in Asia
}

\author{
Benjamin F Katz, Doron S Stember and Harris M Nagler \\ Asian Journal of Andrology (2011) 13, 605-606; doi:10.1038/aja.2010.139
}

In a recently published article in Asian Journal of Andrology, Ho et al. ${ }^{1}$ examine prevalence, attitudes, and treatment patterns related to sexual dysfunction in Asia and contrast them with those of Western society. They highlight the relative paucity of data with respect to erectile dysfunction (ED), premature ejaculation, and hypogonadism in the Asian population. Although the authors make a commendable attempt to characterize sexual dysfunction in Asia, there are multiple factors that complicate interpretation of published prevalence rates. Confounding factors affecting interpretation of these studies include the methodology of identification of the studied cohorts, ages of study participants, survey response rates, definitions of ED, and strategies and lengths of time for data collection.

Both Asia and North America are comprised of diverse urban and rural communities and populations of widely varying educational backgrounds and socioeconomic classes. There is little doubt, therefore, that heterogeneous cultural factors influence reporting and treatment of sexual dysfunction on both continents. The rate of ED in the United States is commonly estimated to be $35 \%-50 \%$ of men aged $40-70$ years. ${ }^{2,3}$ In a review of epidemiologic studies of sexual dysfunction involving Asian countries (including several world studies that included Asian data and allowed for comparison between nations), Lewis demonstrated a wide variation in reported prevalence rates. ${ }^{4}$ For example, men in Thailand had an overall ED rate of $38 \%$, compared with $20 \%$ for China, 15\% for Korea and 2\% for Malaysia. In all studies, however, prevalence rates

Beth Israel Medical Center and the Albert Einstein College of Medicine of Yeshiva University, 1300 Morris Park Avenue, Bronx, NY 10461, USA

Correspondence: Dr H M Nagler (HNagler@chpnet.org) Received: 22 December 2010; Accepted: 4 January 2011 Published online: 6 June 2011 increased with age. There was a 7\%-15\% overall ED prevalence in Asian studies for the 40-49 years age group, which increased to $39 \%-49 \%$ for men aged $60-70$ years. North American, Latin, and Australian populations had similar age-related increases.

The authors of the present review focus on differences in attitudes and treatment between Asian and Western men with ED. They note that many Asian men are 'suffering (sexual dysfunction) in silence' in contrast to men living in Western societies, and suggest that cultural differences related to perception of masculinity may be a root cause. That being said, these same influences affect men and reporting of sexual dysfunction in all cultures. In New York City, we have the privilege to provide care to an exceptionally diverse patient population, including men from across the Asian continent. As in Asia, we routinely encounter patients who are primarily employing alternative and traditional medicine instead of, or along with, so-called "Western" medicine. Men may access these non-traditional therapies because they can be obtained in an anonymous way without divulging embarrassing sexual 'failures'. By definition, alternative medicines have not been subjected to standards of evidencebased demonstration of efficacy and are therefore minimally discussed during medical school and residency training in the United States. Accordingly, North American physicians are typically under-educated with respect to traditional medicine and tend to be skeptical regarding herbal therapy. ${ }^{5}$ Nevertheless, many patients in Asia and the United States rely on herbal medications. Xu and Levine reported that $11-30 \%$ of their patients were taking herbal medicines. They reported that when asked the question, 'How useful do you consider herbal remedies to be in the treatment of your patients?', physicians responded with a median answer of 2 on a 5 point scale, with 5 valued as 'very useful' treatment of patients and 1 as 'not at all useful'. ${ }^{6}$ Given an increasingly diverse society with high levels of continued Asian immigration, we believe that Western physicians would benefit from more concrete and formal training with respect to alternative medicine and that these therapies should be subject to more rigorous evaluation and, perhaps, regulation.

The authors of the review cite cost, availability, lack of documented side effect profile, quality control, and inconsistent effects as important factors related to the ubiquitous utilization of alternative medications in Asia. In Western countries, as in Asia, a myriad of 'natural' products come with claims of improved sexual vitality and growth of the phallus. In a publication by MacKay, L-arginine, yohimbine, Panax ginseng, Maca, Ginkgo biloba, DHEA, and Tribulus terrestris were reviewed for efficacy in treating ED. ${ }^{3}$ The paper's authors concluded that, although evidence-based trials were scarce, the treatments may yield some beneficial effects on penile endothelial tissue, although concern about potential side effects and drug interactions remains. Within the field of urology, there appears to be consensus regarding the need for more clinical and bench research into the efficacy and safety profiles of herbal and traditional medications.

Perhaps the most intriguing disparity that exists between Asian and American cultures is the cultural differences in male health seeking behavior. These differences may persist even among Americans of Asian descent. It has been reported that Asian Americans felt that they were insufficiently involved in the decision making of their own care, perceived that the doctor did not spend enough time with them, and were less likely to agree that 'physicians treat them with respect and dignity' 
neg

Commentary

606

as compared with Caucasian Americans. ${ }^{7}$ In a study examining health beliefs among Chinese students in America, females were more likely than their male counterparts to obtain regular check-ups. The study further delineated the students' perception of their parents in regard to accessing healthcare, and it was reported that the mothers were 'more likely to seek poreventive care and to get regular check-ups than their fathers' ${ }^{8}$ This alarming disparity in gender among Asian Americans reflects concluions reached by the authors of the current review regarding Asians in Asia.

There is clearly a need for healthcare providers to gain the trust of Asian male patients and recognize perceived or real shortcomings regarding mutual respect and shared decision-making responsibilities. Greater understanding of alternative therapies for sexwal dysfunction is important for patients' outcomes and safety. Ongoing efforts by dedicated organizations in Asia, such as the Asia Pacific Society for Sexual Medicine, will be instrumental in removing cultural taboos and facilitating rational therapeutic approaches so that Asian men no longer have to suffer in silence.

1 Ho CCK, Lingam P, Hong GE, Zainuddin ZM. Male sexual dysfunction in Asia. Asian J Androl 2011; 13: 537-542.

2 Layman EO, Park A, Posen RC. Sexual dysfunction in the United States: prevalence and predictors. JAMA 1999; 281: 537-44.
3 MacKay D. Nutrients and botanicals for erectile dysfunction: examining the evidence. Astern Med Rev 2004; 9: 4-16.

4 Lewis RW. Epidemiology of sexual dysfunction in Asia compared to the rest of the world. Asian J Androl 2010; 13: 152-8.

5 Burman BM, Singh BK, Lao L, Singh BB, Ferentz KS et al. Physicians' attitudes toward complementary or alternative medicine: a regional survey. J Am Board Fam Pact 1995; 8: 361-6.

$6 \mathrm{Xu} \mathrm{S}$, Levine M. Medical residents' and students' attitudes towards herbal medicines: a pilot study. Can J Chin Pharmacol 2008; 15: e1-4.

7 Johnson RL, Sha S, Arbelaez JJ, Beach MC, Cooper LA. Racial and ethnic differences in patient perceptions of bias and cultural competence in health care. J Gen Intern Med 2004; 19: 10110.

8 Ray-Mazumder S. Role of gender, insurance status and culture in attitudes and health behavior in a US Chinese student population. Ethn Health 2001; 6: 197-209.

Asian Journal of Andrology 